\title{
Correction to: The impact of clinical pharmacy services in Nepal in the context of current health policy: a systematic review
}

\author{
Reyaj Mikrani ${ }^{1} \cdot$ Muhammad Naveed $^{2} \cdot$ Aman Mikrani $^{3} \cdot$ Sufia Yasmeen ${ }^{1} \cdot$ MD. Akabar $^{4} \cdot$ Zhou Xiaohui $^{1,5,6}{ }^{\text {(D) }}$
}

Published online: 22 May 2019

(C) Springer-Verlag GmbH Germany, part of Springer Nature 2019

\section{Correction to: J Public Health (Berl.): From Theory to Practice https://doi.org/10.1007/s10389-019-01042-y}

The Fig. 1 was incompletely filled. The correction and corresponding data are shown below.

Addition to Fig. 1 "Records identified through database searching": Cochrane library $(n=6)$.

Publisher's note Springer Nature remains neutral with regard to jurisdictional claims in published maps and institutional affiliations.

The online version of the original article can be found at https://doi.org/ 10.1007/s10389-019-01042-y

Zhou Xiaohui

zhxh@cpu.edu.cn

1 Department of Clinical Pharmacy, School of Basic Medicine and Clinical Pharmacy, China Pharmaceutical University, \#639 Longmian Avenue, Jiangning District, Nanjing 211198, People's Republic of China

2 Department of Clinical Pharmacology, School of Pharmacy, Nanjing Medical University, \#101 Longmian Avenue, Jiangning District, Nanjing 211166, People's Republic of China

3 Department of Finance, College of Economics and Management, Nanjing University of Aeronautics and Astronautics, \#29 Jiangjun Avenue, Jiangning District, Nanjing 211100, People's Republic of China
4 Department of Pharmaceutics, School of Pharmacy, China Pharmaceutical University, \#639 Longmian Avenue, Jiangning District, Nanjing 211198, People's Republic of China

5 Department of Cardiothoracic Surgery, Zhongda Hospital affiliated to Southeast University, \#87 Dingjiaqiao, Jiangning District, Nanjing 210017, People's Republic of China

6 Department of Heart Surgery, Nanjing Shuiximen Hospital, \#283 Shuiximen Avenue, Jiangning District, Nanjing 210017, People's Republic of China 

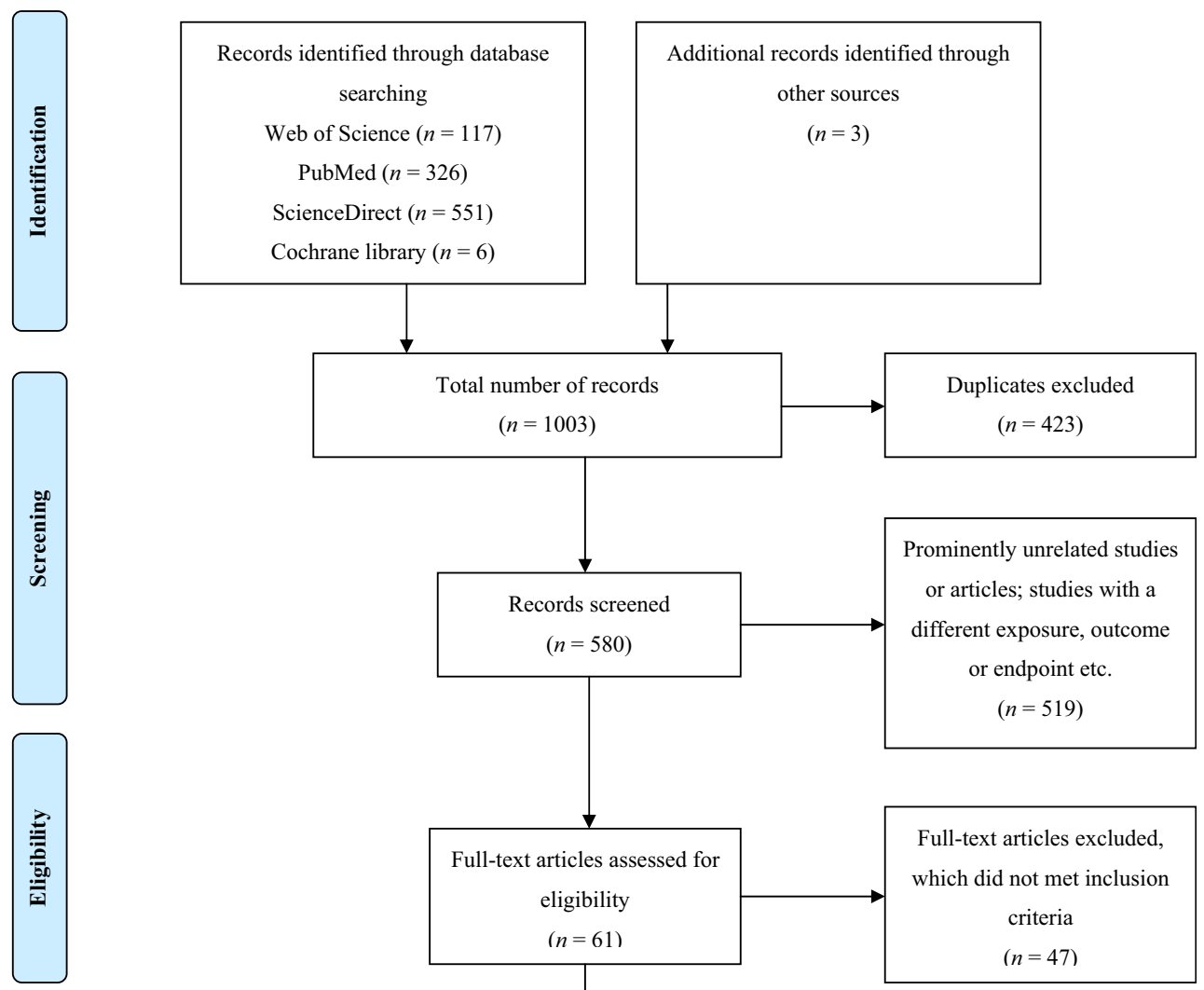

Full-text articles excluded,

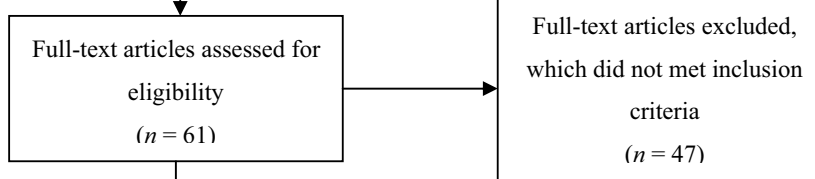

节

Studies included in

qualitative synthesis

$(n=14)$

Fig. 1 Preferred Reporting Items for Systematic Reviews and Meta-Analyses (PRISMA) flow diagram of the study process 